\title{
Rescue Thrombolysis Followed by Salvage Percutaneous Coronary Intervention for the Treatment of Inferior ST Elevation Myocardial Infarction Combined with Cardiogenic Shock in a Patient with an Anomalous Right Coronary Artery Origin
}

Ji-Hyuck Rhee and Sung Woo Kwon*

Division of Cardiology, Department of Internal Medicine, Yongin Severance Hospital, Yonsei University College of Medicine, Yongin, Republic of Korea

\begin{abstract}
We report a case of rescue thrombolysis followed by salvage percutaneous coronary intervention (PCI) for the treatment of inferior ST elevation myocardial infarction (STEMI) combined with cardiogenic shock in a patient with an anomalous right coronary artery (RCA) origin. When it is not feasible to perform primary $\mathrm{PCl}$ due to the anomalous origin of the RCA, rescue thrombolysis can be an optional treatment strategy that may allow sufficient time to perform facilitated $\mathrm{PCl}$ in a STEMI patient with hemodynamic instability.
\end{abstract}

Keywords: Thrombolytic therapy; Percutaneous coronary intervention; Coronary vessel anomalies; Myocardial infarction; Cardiogenic shock

\section{Introduction}

Performing primary percutaneous coronary intervention (PCI) in a ST elevation myocardial infarction (STEMI) patient with hemodynamic instability may be challenging since conventional diagnostic and guiding catheters are generally designed to approach coronary arteries with normal origins [1-3]. In this case, thrombolytic therapy can be an alternative revascularization strategy for the treatment of patient with STEMI to gain time. However, some of the STEMI patients who receive thrombolytic therapy fail to achieve patency of the infarct-related coronary artery [4]. Under these circumstances, performing salvage PCI to restore coronary blood flow soon after the thrombolysis fails seems to be necessary [5]. Herein, we report a case of rescue thrombolysis followed by salvage PCI for the treatment of inferior STEMI combined cardiogenic shock in a patient with anomalous right coronary artery (RCA) origin. When it is not feasible to perform primary PCI due to the anomalous origin of the RCA, rescue thrombolysis can be an optional treatment strategy that may allow sufficient time to perform facilitated PCI in a STEMI patient with hemodynamic instability.

\section{Case Report}

A 39-year-old male patient was admitted to our emergency department with sudden onset of severe chest pain for 30 minutes. He had no relevant past medical history. Physical examination revealed a heart rate of 45 beats/min and blood pressure of $78 / 45 \mathrm{mmHg}$, which indicated cardiogenic shock. Auscultation showed clear lung sounds, bradycardia and no murmur or gallop. Initial 12-lead electrocardiography revealed ST segment elevation in the inferior leads with complete atrioventricular block (CAVB) suggestive of inferior wall STEMI (Figure 1a). Intravenous volume infusion followed by aspirin, clopidogrel loading and intravenous heparinization was performed immediately. Fortunately, the heart rate as well as the blood pressure increased to the normal range (blood pressure 114/76 $\mathrm{mmHg}$ and heart rate 81 beats/min). Follow-up electrocardiography revealed resolution of the CAVB but the ST segment elevation still remained in the inferior leads (Figure 1b). So, he was transferred to the catheterization laboratory for primary PCI. Because of the hemodynamic instability, the right common femoral vein was punctured and a transvenous temporary pacemaker was prepared in case of CAVB development. Then, the right common femoral artery was punctured to perform invasive coronary angiography. The left coronary artery was approached by a $5 \mathrm{~F} \mathrm{Judkins} \mathrm{left}$ 4 diagnostic catheters and it revealed no significant luminal narrowing at the left anterior descending coronary artery and the left circumflex coronary artery. An attempt was made to engage the RCA with a $5 \mathrm{~F}$ Judkins right 4 diagnostic catheter but this failed. We could not find the RCA ostium. So, we immediately performed an aortogram with a $5 \mathrm{~F}$ pigtail catheter and found that the RCA was originated from the left sinus of Valsalva- below the sinotubular plane between the midline and origin of the left coronary artery. We tried to engage the RCA with a $6 \mathrm{~F}$ Amplatzer 1 guide catheter but it was not feasible. At that time, the patient suffered more severe chest pain, and his blood pressure got lower. Intravenous fluids were administered followed by an intravenous dobutamine infusion. We knew that we did not have much time. We could have re-tried RCA engagement using an Amplatzer catheter, but we could not be sure of having success with the RCA engagement right away. So, we decided to perform rescue thrombolysis in order to gain time. Thrombolytic therapy was administered with Alteplase, the recombinant tissue plasminogen activator (t-PA) (total dose 100 $\mathrm{mg}$; bolus of $15 \mathrm{mg} \mathrm{IV}$, followed by $50 \mathrm{mg}$ for 30 minutes and $35 \mathrm{mg}$ for the next 60 minutes). ST segment resolution was noted during the $\mathrm{t}$-PA administration. The patient's chest symptom resolved as well and the blood pressure rose slowly. Accelerated idioventricular rhythm was present for about 10 minutes during the t-PA infusion. After rescue thrombolysis; we planned to perform adjunctive PCI (after 3 hours, but

*Corresponding author: Sung Woo Kwon, Clinical Assistant Professor, Division of Cardiology, Department of Internal Medicine, Yongin Severance Hospital, Yonse University College of Medicine, 225 Geumhak-no, Cheoin-gu, Yongin-si, Gyeonggido, 449-930, Republic of Korea, Tel: (82) 31-331-8723; Fax: (82) 31-331-8722; E-mail: mdsungwoo@yuhs.ac

Received November 13, 2013; Accepted November 27, 2013; Published December 04, 2013

Citation: Rhee JH, Kwon SW (2013) Rescue Thrombolysis Followed by Salvage Percutaneous Coronary Intervention for the Treatment of Inferior ST Elevation Myocardial Infarction Combined with Cardiogenic Shock in a Patient with an Anomalous Right Coronary Artery Origin. J Cardiovasc Dis Diagn 2: 133. doi:10.4172/2329-9517.1000133

Copyright: ( $2013 \mathrm{Rhee} \mathrm{JH}$, et al. This is an open-access article distributed unde the terms of the Creative Commons Attribution License, which permits unrestricted use, distribution, and reproduction in any medium, provided the original author and source are credited. 
Citation: Rhee JH, Kwon SW (2013) Rescue Thrombolysis Followed by Salvage Percutaneous Coronary Intervention for the Treatment of Inferior ST Elevation Myocardial Infarction Combined with Cardiogenic Shock in a Patient with an Anomalous Right Coronary Artery Origin. J Cardiovasc Dis Diagn 2: 133. doi:10.4172/2329-9517.1000133

(a)

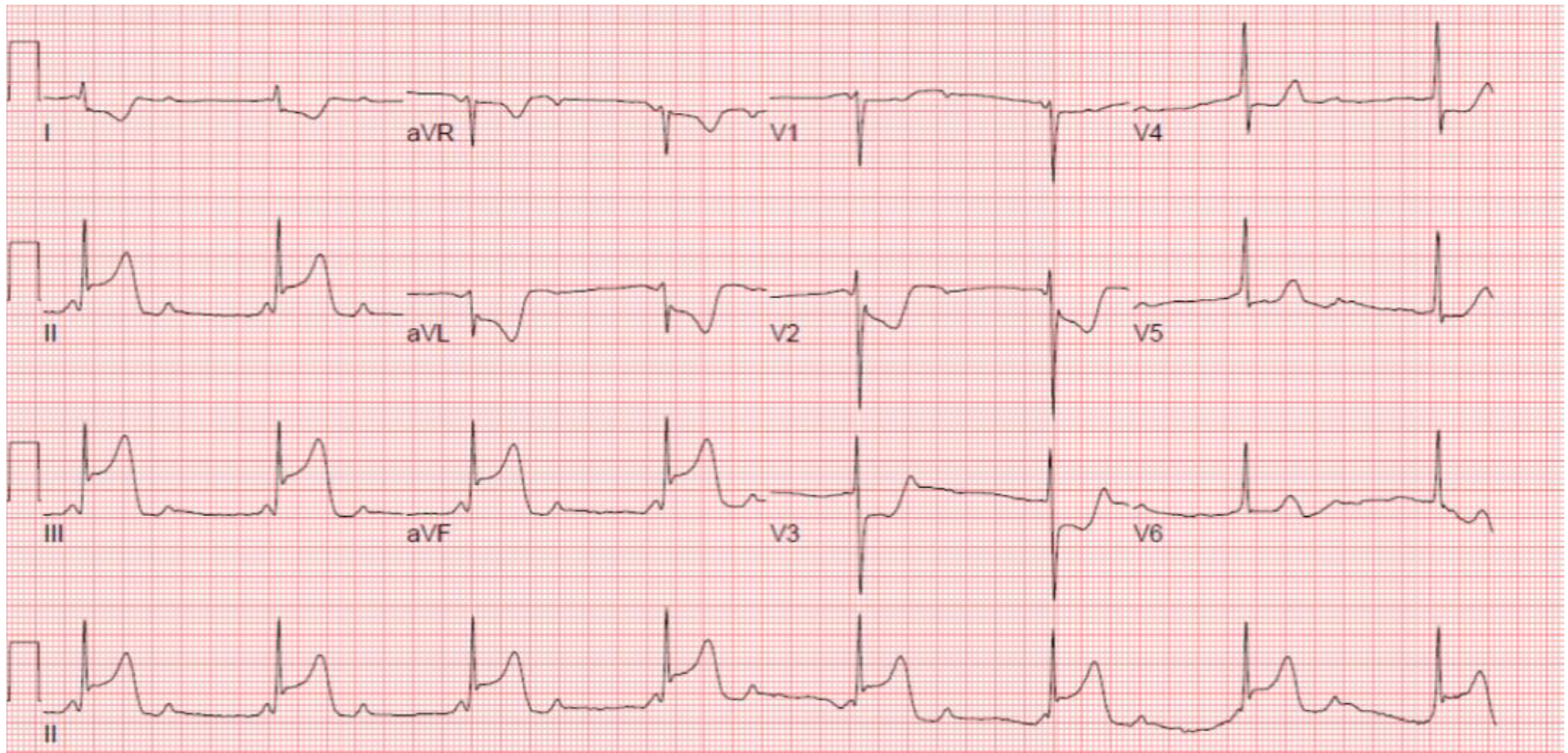

(b)

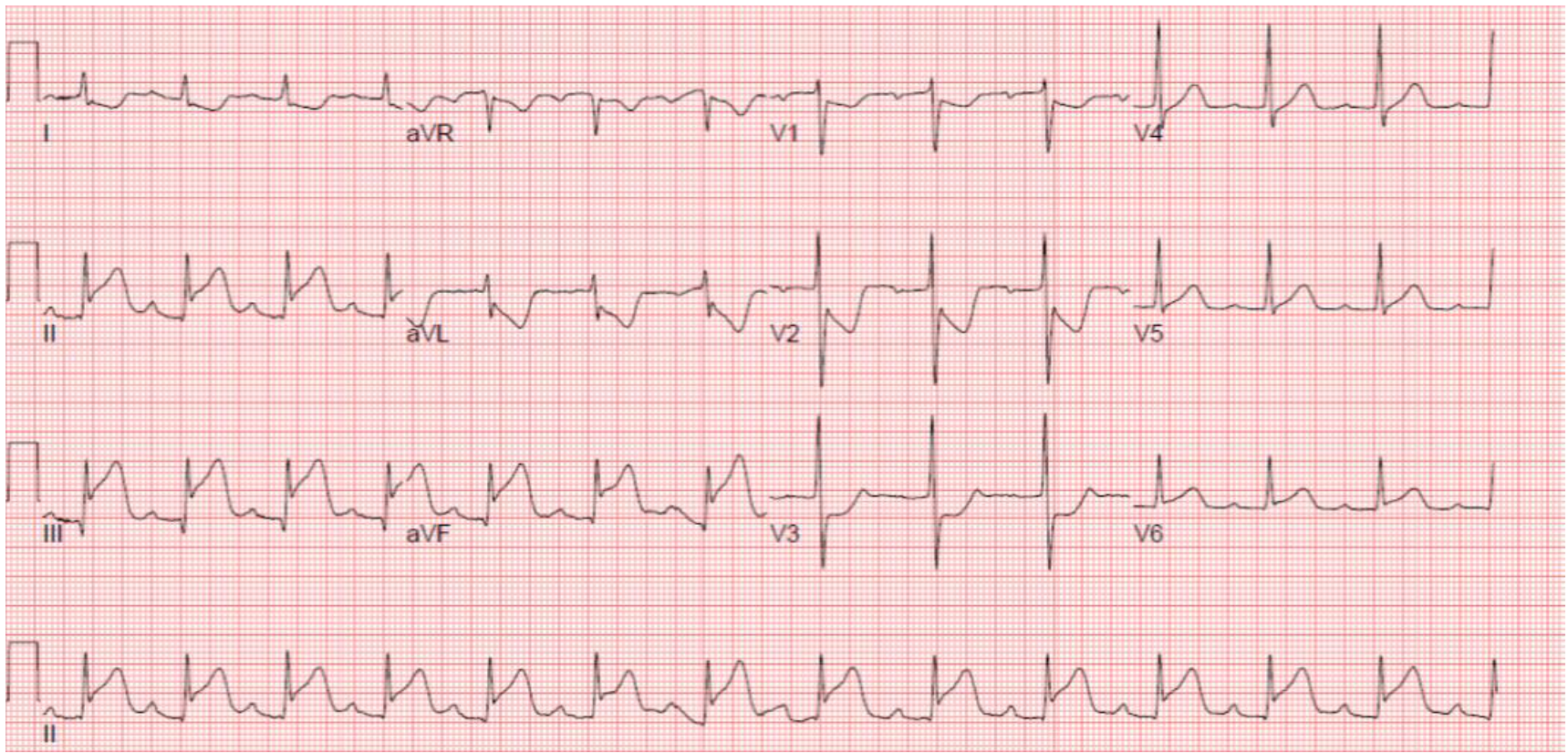

Figure 1: Initial electrocardiography of the patient revealed (a) complete atrioventricular block (CAVB) with marked ST segment elevation in the inferior leads and (b) resolution of the CAVB but ST segment elevation still remained in the inferior leads after intravenous fluid infusion followed by aspirin, clopidogrel loading and intravenous heparinization.

within 24 hours after the thrombolysis). However, about 10 minutes after the rescue thrombolysis, the patient suffered from chest pain again, and ST segment elevation was noted on electrocardiographic monitoring. So, we decided to perform facilitated PCI, since the RCA was thought to be re-occluded. We tried to engage the RCA with a 6F Amplatzer 1 guide catheter, and it engaged successfully. An RCA angiogram revealed total occlusion of the mid-RCA with Thrombolysis in Myocardial Infarction (TIMI) grade 0 flow (Figure 2a). We introduced a 0.014 inch J-curved Fielder wire and passed the lesion. Predilatation was performed with an Ikazuchi $2.5 \times 15 \mathrm{~mm}$ balloon at the mid-RCA. Two Resolute Integrity stents $3.0 \times 26 \mathrm{~mm}$ and $3.0 \times 18$ $\mathrm{mm}$ were implanted sequentially at the mid-RCA. The final RCA angiogram showed revascularization of the infarct-related RCA with TIMI grade 3 flow (Figure 2b). The patient's chest symptom as well as 
Citation: Rhee JH, Kwon SW (2013) Rescue Thrombolysis Followed by Salvage Percutaneous Coronary Intervention for the Treatment of Inferior ST Elevation Myocardial Infarction Combined with Cardiogenic Shock in a Patient with an Anomalous Right Coronary Artery Origin. J Cardiovasc Dis Diagn 2: 133. doi:10.4172/2329-9517.1000133

Page 3 of 3

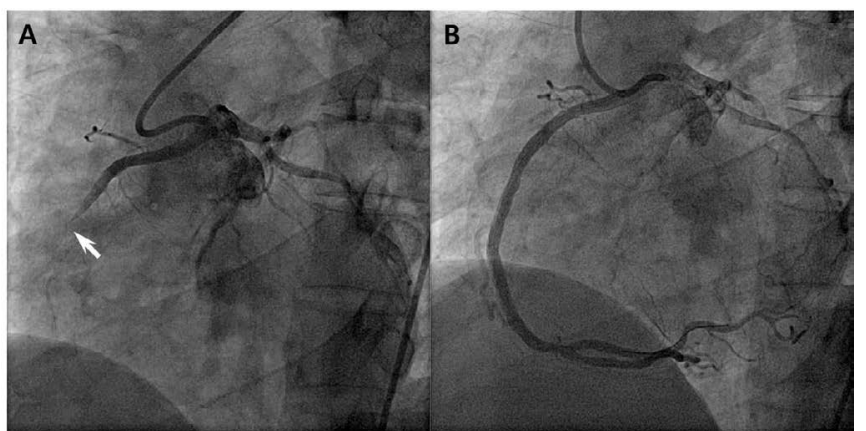

Arrow indicates totally occluded lesion of the anomalous RCA.

Figure 2: Angiographic images of the anomalous right coronary artery (RCA) (a) before and (b) after facilitated $\mathrm{PCl}$ (left anterior oblique view).

the ST segment elevation on electrocardiographic monitoring resolved completely. The patient's blood pressure and heart rate were stable and he was sent to the intensive care unit for further management. Initial laboratory analysis revealed that the creatine kinase (CK) was $114 \mathrm{U} / \mathrm{L}$ (normal values: $24-170 \mathrm{U} / \mathrm{L}$ ), CK-MB, $1.4 \mathrm{mcg} / \mathrm{mL}$ (normal values: 0-6.6 mcg/mL), troponin I, $0.017 \mathrm{ng} / \mathrm{mL}$ (normal values: $<0.028 \mathrm{ng} /$ $\mathrm{mL}$ ), and brain natriuretic peptide, $10.0 \mathrm{pg} / \mathrm{mL}$ (normal values: $0-100$ $\mathrm{pg} / \mathrm{mL}$ ). Transthoracic echocardiography was performed 6 hours after the facilitated PCI. Transthoracic echocardiography revealed reduced left ventricular (LV) systolic function with an ejection fraction of $47 \%$. Regional wall motion abnormalities were also noted, showing akinesis of the inferior wall from the basal segment to the apical segment, the posterolateral wall from the mid-LV segment to the apical segment, and severe hypokinesis of the inferoseptal wall from the basal segment to the apical segment. During admission, the peak levels of cardiac biomarkers were CK >2000 U/L (normal values: 24-170 U/L); CK-MB $87.0 \mathrm{mcg} / \mathrm{mL}$ (normal values: $0-6.6 \mathrm{mcg} / \mathrm{mL}$ ), and troponin $\mathrm{I}>50.0$ $\mathrm{ng} / \mathrm{mL}$ (normal values: $<0.028 \mathrm{ng} / \mathrm{mL}$ ). Fasting glucose level was 129 $\mathrm{mg} / \mathrm{dL}$ and post-prandial glucose level was $265 \mathrm{mg} / \mathrm{dL}$. In addition, the glycated hemoglobin level was $7.5 \%$. So, under the diagnosis of new onset type 2 diabetes, we started anti-diabetic medication with biguanides. Lipid profiles consisting of total cholesterol, triglycerides, high density lipoprotein cholesterol, and low density lipoprotein cholesterol were $217,151,45$, and $137 \mathrm{mg} / \mathrm{dL}$, respectively. Therefore, we also started lipid lowering therapy with atorvastatin $40 \mathrm{mg}$. The patient was stable during the rest of his admission, and afterwards, he was discharged with medications.

\section{Discussion}

Along with primary PCI, thrombolytic therapy is an alternative revascularization strategy for the treatment of patients with STEMI. However, $30 \%$ to $50 \%$ of STEMI patients fail to achieve patency of the infarct-related coronary artery at $90 \mathrm{~min}$ after the onset of thrombolytic therapy [4]. Under these circumstances, facilitated PCI may play a crucial role inrestoring the blood flow of the re-occluded infarctrelated coronary artery. A prior study demonstrated that salvage PCI performed early after failed thrombolysis seems to be as effective and safe as primary PCI [5]. Moreover, another compelling study revealed the virtue of salvage PCI as a treatment strategy since it is beneficial when compared to repeat thrombolysis as well as conservative therapy, in terms of prognosis [6]. Therefore, performing salvage PCI to restore coronary blood flow soon afterthe thrombolysis fails seems to be necessary. When performing salvages PCI, bleeding complications are the main concern. However, earlier studies revealed that thebleeding complication rate in patients undergoing salvage PCI was comparable to those undergoing primary PCI or delayed PCI after thrombolysis $[6,7]$.

Although coronary arteries of anomalous origin are uncommon, with an incidence of $0.2-1.2 \%$ of patients who undergo PCI, they may be challenging since conventional diagnostic and guiding catheters are generally designed to approach coronary arteries with normal origins [1-3]. An anomalous origin of the RCA from the left sinus of Valsalva has been reported in 6-27\% of patients with coronary anomalies [8]. Intriguingly, a previous study suggested that the presence of this anomaly may be associated with an increased predisposition to developing coronary atherosclerotic disease [9].

We report a case of rescue thrombolysis followed by salvage PCI for the treatment of inferior STEMI combined with cardiogenic shock in a patient with an anomalous RCA origin. Time does not matter for performing an 'elective PCI' for treating the patient with ananomalous RCA origin since the patient is hemodynamically stable during the procedure. However, time matters for performing a 'primary $\mathrm{PCI}$ for treating a patient with an anomalous RCA origin presenting with STEMI and cardiogenic shock, due to the hemodynamic instability. Our case illustrates that rescue thrombolysis can be an optional treatment strategy that may allow sufficient time for the performance of facilitated PCI, when it is not feasible to perform primary PCI due to an anomalous origin of a coronary artery.

\section{References}

1. Kimbiris D, Iskandrian AS, Segal BL, Bemis CE (1978) Anomalous aortic origin of coronary arteries. Circulation 58: 606-615

2. Click RL, Holmes DR Jr, Vlietstra RE, Kosinski AS, Kronmal RA (1989) Anomalous coronary arteries: location, degree of atherosclerosis and effect on survival--a report from the Coronary Artery Surgery Study. J Am Coll Cardiol 13: $531-537$

3. Park JH, Kwon NH, Kim JH, Ko YJ, Ryu SH, et al. (2013) Prevalence of congenital coronary artery anomalies of korean men detected by coronary computed tomography. Korean Circ J 43: 7-12.

4. (1993) An international randomized trial comparing four thrombolytic strategies for acute myocardial infarction. The GUSTO investigators. N Engl J Med 329 673-682.

5. Poloński L, Gasior M, Wasilewski J, Wilczek K, Wnek A, et al. (2003) Outcomes of primary coronary angioplasty and angioplasty after initial thrombolysis in the treatment of 374 consecutive patients with acute myocardial infarction. Am Heart J 145: 855-861.

6. Gershlick AH, Stephens-Lloyd A, Hughes S, Abrams KR, Stevens SE, et al. (2005) Rescue angioplasty after failed thrombolytic therapy for acute myocardial infarction. N Engl J Med 353: 2758-2768.

7. Balachandran KP, Miller J, Pell AC, Vallance BD, Oldroyd KG (2002) Rescue percutaneous coronary intervention for failed thrombolysis: results from a district general hospital. Postgrad Med J 78: 330-334.

8. Sarkar K, Sharma SK, Kini AS (2009) Catheter selection for coronary angiography and intervention in anomalous right coronary arteries. J Interv Cardiol 22: 234-239.

9. Jim MH, Siu CW, Ho HH, Miu R, Lee SW (2004) Anomalous origin of the right coronary artery from the left coronary sinus is associated with early development of coronary artery disease. J Invasive Cardiol 16: 466-468.

Citation: Rhee JH, Kwon SW (2013) Rescue Thrombolysis Followed by Salvage Percutaneous Coronary Intervention for the Treatment of Inferior ST Elevation Myocardial Infarction Combined with Cardiogenic Shock in a Patient with an Anomalous Right Coronary Artery Origin. J Cardiovasc Dis Diagn 2 133. doi:10.4172/2329-9517.1000133 